\title{
Huellas de la primera publicación en un docente de inglés: un relato de vida
}

Primer semestre de 2019 - pp. 161-175

The Traces of a First

Publication in an English

Teacher: A Life Story
A marca indelével da primeira publicação em um professor de inglês: um relato de vida

Melba Libia Cárdenas* Orcid: https://orcid.org/0000-0003-0028-2506

\section{Para citar este artículo}

\section{Cárdenas, M. L. (2019). Huellas de la primera publicación en un docente de inglés: un relato de vida. Folios, 49, 161-175. \\ doi: 10.17227/Folios.49-9403}

Magíster en TESOL y doctoranda en Educación. Profesora del Departamento de Lenguas Extranjeras y Directora de Relaciones Exteriores, Universidad Nacional de Colombia.

Correo electrónico: mlcardenasb@unal.edu.co 


\section{Resumen}

Este artículo se centra en el relato de vida de un docente, participante en un estudio de caso de corte etnográfico que busca conocer, entre otros aspectos, el papel que atribuyen profesores de inglés a la publicación de su primer artículo en una revista académica o científica, en su ciclo profesional. Para la recolección de información se emplearon entrevistas a profundidad y evidencias documentales. En primer lugar, se presentan los elementos conceptuales que guiaron la aplicación del enfoque narrativo en lo concerniente a los relatos de vida. Luego se describen el contexto de la investigación y el proceso metodológico. A continuación, y puesto que se optó por el análisis narrativo, se incluye el relato de vida de un docente de inglés, ilustrado a partir de su trayectoria como escritor. El relato muestra el papel de la escritura académica y la publicación de sus trabajos en la vida de los profesores, así como algunas implicaciones para fomentar un rol activo en sus comunidades académicas.

\section{Palabras clave}

escritura académica; investigación narrativa; publicación científica; relato de vida

\section{Abstract}

This paper focuses on the life story of a teacher who took part in an ethnographic case study that aims to learn, among other things, about the role that English teachers give to the publication of their first paper in an academic or scientific journal in their career. In-depth interviews and documentary evidence were used to gather information. First, the paper presents the conceptual elements that guided the implementation of the narrative approach regarding life stories. Then, it describes the context of the research and the methodological process. Next, and since narrative analysis was chosen, the life story of an English teacher is included, illustrated from his career as a writer. The story shows the role of academic writing and of the publication of their works in the lives of teachers, as well as some implications for fostering an active role in their academic communities.

\section{Keywords}

academic writing; narrative research; scientific publication; life story

\section{Resumo}

Este artigo está focado no relato de vida de um professor, participante em um estudo de caso de tipo etnográfico que visa conhecer, entre outros aspectos, o papel atribuído pelos professores de inglês à publicação do seu primeiro artigo em uma revista acadêmica ou científica, em sua vida profissional. Para a coleta de informações utilizamos entrevistas a profundidade e evidências documentais. Em primeiro lugar, apresentamos os elementos conceptuais que guiaram a aplicação do enfoque narrativo no relacionado com os relatos de vida. Em seguida, describimos o contexto da pesquisa e o processo metodológico. A continuação, e já que optamos pela análise narrativa, apresentamos o relato de vida de um professor de inglês, construído desde sua trajetória como escritor. 0 relato evidencia o papel da escrita acadêmica e a publicação de seus trabalhos na vida dos professores, assim como algumas implicações para fomentar o desempenho de um papel ativo em suas comunidades académicas.

\section{Palavras-chave}

escrita acadêmica; pesquisa narrativa; publicação científica; relato de vida 


\section{Introducción}

La publicación de resultados de investigación y del conocimiento generado en el país es un factor importante en las políticas colombianas de ciencia y tecnología, pues se espera, entre otras metas, asegurar una mayor visibilidad internacional principalmente a través de revistas científicas o académicas (Colciencias, 2016). En Colombia, al igual que en muchos otros países de América Latina, ha tomado fuerza la pertinencia de que los docentes universitarios investiguen y publiquen, preferiblemente en revistas indizadas (Delgado, 2011). Esto, a su vez, ha mostrado la necesidad de fomentar la escritura académica y el desarrollo de competencias investigativas en la formación inicial y permanente de los profesores de lenguas. Ante estas circunstancias, merece preguntarse si es posible que los docentes atiendan simultánea y efectivamente los diversos frentes académicos que conllevan su ejercicio profesional (Cortés, Cárdenas y Nieto, 2013; Oviedo, 2009; Pérez, 2010) y si tienen la oportunidad de incursionar en foros académicos establecidos, donde tradicionalmente se visibilizan trabajos de grupos de investigación de renombre o con un arraigo primordialmente en universidades (Cárdenas, 2014).

Por otra parte, y en relación con quienes logran publicar sus escritos en revistas científicas o académicas, es preciso conocer acerca de sus experiencias como escritores y las huellas que esto deja en su vida profesional. Este aspecto, abordado a partir del relato de vida de un docente, constituye el foco de atención del presente artículo. Por tanto, se han tenido en cuenta aspectos de la investigación narrativa.

Partimos del supuesto de que la narración es "la única forma lingüística adecuada para mostrar la existencia humana como acción contextualizada. Las descripciones narrativas muestran que la actividad humana es una implicación en el mundo con propósito" (Polkinghorne, 1995, p. 5).

La investigación narrativa también denominada biográfica o biográfico-narrativa "es el estudio de las formas en que los seres humanos experimentamos el mundo" (Connelly y Clandinin, 1990, p. 6) y, por tanto, incluye el uso de diversos tipos de textos para aproximarnos a la vida de los sujetos: diarios, relatos, evidencias documentales, correspondencia, historias de vida, entre otros (Barkhuizen y Wette, 2008; Bolívar, 2002; Muñoz, 2012; Polkinghorne, 1995, 2007). Como herramientas de investigación, dichos textos ofrecen amplias "posibilidades para explorar de forma humanista unas experiencias sociales concretas" (Plummer, 1989, p. 40). Se trata esencialmente de darle sentido a las situaciones propias de los sujetos. Esto es posible gracias a relatos o textos narrativos, a través de los cuales nos acercamos a diversos discursos y, como tejedores, elaboramos interpretaciones, "mecanismos de producción de significación y de elaboración simbólica de la realidad" (Muñoz, 2012, p. 161).

En este escrito se presenta el relato de vida de un docente de inglés, donde se evidencia el papel que atribuye a la publicación de su primer artículo en la revista Profile: Issues in Teachers' Professional Development (en adelante, Profile), editada en Colombia, en su ciclo profesional. A continuación se describen el contexto de la investigación y los elementos metodológicos que guiaron la aplicación del enfoque narrativo en lo concerniente a los relatos de vida. Luego se incluye el citado relato, elaborado a partir de la información recolectada. Finalmente, se resaltan algunos elementos que surgen de su análisis, con el propósito de establecer el significado de las experiencias del informante como escritor y vislumbrar implicaciones tanto para la formación del profesorado, como para la divulgación del conocimiento en publicaciones académicas.

\section{El relato de vida}

Es importante precisar, conforme al uso que se les da en las literaturas anglosajona, francesa y española, el sentido de relato de vida (life story, récit de vie o narración) e historia de vida (life history o histoire de vie). Denzin (1970) emplea el término relato de vida para la historia de una vida tal como la rememora quien la ha vivido, mientras que propone reservar la historia de vida para los estudios de casos sobre una persona determinada. Así, la historia de vida incluye, además de su propio relato de vida, otros documentos como la historia clínica, el expediente 
judicial, las pruebas psicológicas, los testimonios de allegados, entre otros.

Por su parte, Bolívar y Domingo (2006) instan a distinguir entre:

1. life story, récit de vie o relato de vida: como la narración de una vida tal como la persona la ha vivido o cuenta.

2. life history, histoire de vie o historia de vida: o conjunto del anterior concepto y las elaboraciones externas de biógrafos o investigadores, así como los registros, entrevistas, etc., que permiten validar esta narración y/o historia (par. 112).

Se entiende el relato de vida como la historia de una persona, tal y como la cuenta quien la ha experimentado (Bertaux, 1999, 2005, 2009; Denzin, 1970). El relato de vida privilegia la voz del sujeto, de tal manera que pueda hablar ampliamente sobre sus vivencias, sin las restricciones que acarrean el uso de otros dispositivos o instrumentos de recogida de datos. Esto "permite que el investigador trabaje con las resonancias personales que tienen en él las palabras y actitudes del narrador" (Legrand, 1999, p. 1).

Para Polkinghorne (1995), un relato es la producción especial de una clase de discurso. En él encontramos un comienzo, un intermedio y un final, así como componentes fundamentales.

Como establece Scholes (1982, en Carter, 1993), en un relato hay al menos tres elementos básicos: (a) una situación que encierra algún conflicto o lucha, (b) un protagonista que se involucra en la situación con un propósito, y (c) una secuencia con una causalidad implícita (una trama), durante la cual se resuelve el conflicto. (Mendieta, 2013, p. 137).

Un narrador o investigador reconstruye el relato a través de un discurso que conecta diversos eventos, incidentes y acciones llevadas a cabo por algunos personajes en circunstancias contextuales cambiantes que, a su vez, generan la reacción de los individuos (Bertaux, 2005; Bolívar, 2002; Ricoeur, 1995, 1999). En esa búsqueda de conexiones "el proceso de (re)creación del yo en la investigación biográfica se convierte en un elemento vertebral anudado a una particular manera de entender la temporalidad (tiempo-memoria) y el relato de sí (testimonio-narración)" (Argüello, 2014, p. 297). Por tanto, el relato de vida constituye "una herramienta incomparable de acceso a lo vivido subjetivamente, y la riqueza de sus contenidos es una fuente de hipótesis inagotable" (Bertaux, 1999, p. 2).

Puesto que no hay una verdad total en los documentos humanos, sino perspectivas, se reconoce que "todos los relatos, incluso los científicos, son narrados desde un punto de vista" (Plummer, 1989, p. 69). Asimismo, se genera un interés dual por lo subjetivo y lo objetivo. El mismo autor resalta que hay preferencia por los marginados y los perdedores, para lo cual se $d a$ la palabra a los que no siempre son escuchados, para avanzar hacia una comprensión y un realineamiento de la acción (Plummer, 1989, p. 70).

La característica principal del relato de vida, según Bertaux (2005, p. 12) es "constituir un intento de descripción de la estructura diacrónica del recorrido vivencial, característica que lo distingue radicalmente de las demás formas (no narrativas) de entrevista”. En esta misma línea de pensamiento, Pujadas (1992, p. 33) destaca el uso de los documentos personales como "medio de formulación emic" que ilustran fenómenos y posibilitan el trabajo con otros medios, fundamentalmente etnográficos.

\section{Contexto de la investigación}

Las publicaciones en revistas cumplen un papel preponderante en la promoción de los docentes colombianos en la carrera profesoral, así como en la evaluación de las universidades. Asimismo, inciden en la obtención de incentivos como becas para posgrados, en el acceso a plazas laborales, en el empoderamiento y reconocimiento del docente como investigador (Cárdenas, 2003, 2014; Cárdenas y Nieto, 2010; Cárdenas, González y Álvarez, 2010), en la clasificación de los grupos de investigación colombianos y en la categorización de los investigadores como sénior, asociado o júnior (Colciencias, 2015).

Si bien no resulta fácil para un escritor novel o con poca experiencia investigativa publicar en revistas científicas o académicas editadas en el país, 
las oportunidades para lograrlo son actualmente amplias y diversas a causa del incremento del número de ellas en las dos últimas décadas. Según el último estudio del Observatorio Colombiano de Ciencia y Tecnología, de la totalidad (515) de las revistas clasificadas en el Índice Bibliográfico Nacional (IBN) Publindex, 208 son del área de ciencias sociales y 71 de humanidades (Lucio-Arias et al., 2015). ${ }^{1}$ De este último grupo, 31 se centran en el campo de la educación, 17 abordan temáticas de lenguas y literatura y de ellas, tres publican completamente en lengua inglesa -Profile es una de ellas-. La revista fomenta la divulgación de investigaciones, innovaciones y reflexiones sobre la enseñanza de la lengua inglesa, la formación y el desarrollo del profesorado en el área. La publicación es auspiciada por la Universidad Nacional de Colombia y circula con una periodicidad semestral, en versiones impresa y electrónica.

\section{Método}

Se siguió el enfoque narrativo en lo ateniente a los relatos de vida, los cuales se elaboraron durante la fase interpretativa de un estudio de caso de corte etnográfico (Sabirón, 2007). En ese estudio macro participan doce autores (cinco de ellos son pares evaluadores de la revista y tres han publicado al menos un artículo en ella). En cuanto a su nacionalidad, tenemos: dos de España, uno de Estados Unidos, uno de México, uno del Reino Unido y siete de Colombia. Diez de los doce autores son docentes universitarios y dos profesoras laboran en escuelas o colegios. El presente artículo se centra en el relato de vida de un docente universitario colombiano.

En su elaboración se siguieron las siete etapas formuladas por Creswell (2013) para la investigación narrativa: identificación del fenómeno, selección de un individuo, recopilación de relatos, reformulación o narración del relato del individuo, colaboración

1 El Servicio de Indexación-Publindex, administrado por Colciencias, realizó la Convocatoria No. 768 de 2016 y publicó los resultados del Índice Bibliográfico Nacional (IBN) Publindex en septiembre de 2017. Allí se hallan solo 244 revistas clasificadas. Está pendiente la publicación del listado final, por cuanto aún falta incluir aquellas revistas que presentaron reclamaciones y, luego de la revisión de la evaluación realizada por Publindex, fueron clasificadas. con el relator participante, escritura de un relato y validación de la exactitud del reporte. Estos siete estadios no implican una ruta lineal, pero sí engloban procesos metodológicos acordes con los fundamentos del enfoque investigativo.

En relación con los instrumentos para la recogida de datos, se emplearon entrevistas a profundidad y evidencias documentales. Estas últimas fueron fuentes complementarias o secundarias e incluyen los datos recopilados en las hojas de vida de todos los participantes del estudio macro y comunicaciones establecidas con ellos por correo electrónico. El material que se recoge en las entrevistas fue sumamente rico. Como apunta Molano (1998, p. 104), en parte está coloreado y determinado por la relación entre quien investiga y los participantes. Estos colores o matices otorgan singularidad a los relatos, por cuanto en su elaboración se mantuvo un respeto absoluto por el lenguaje empleado por los entrevistados.

Por otra parte, cabe señalar que, por tratarse de un estudio de corte etnográfico, la editora de la revista -autora de este texto y quien realiza la investigación- ha tenido el papel de observadora participante. A este respecto, merece señalarse que el relato de vida queda aquí subsumido en la observación participante, por cuanto en el relato vivenciado, documentado y narrado (observado) interviene el propio observador (participante) (Arraiz y Sabirón, 2011). Así, el investigador es un inductor de la relación entre el sujeto y la realidad social que evoca.

El relato de vida de Yesid (seudónimo seleccionado por el participante) se elaboró principalmente a partir de tres entrevistas a profundidad (la primera, en septiembre 16 de 2013; la segunda, en enero 27 de 2014, y la tercera, en diciembre 23 de 2016). Además, se tuvieron en cuenta datos de su curriculum vitae y de correos electrónicos que hemos intercambiado.

Para el análisis de la información recolectada se siguió la modalidad narrativa. $\mathrm{Al}$ respecto, vale la pena definir los dos modos de conocimiento señalados por J. Bruner (1998): narrativo y paradigmático. 
El primero es hermenéutico; centra su atención en las vicisitudes de la intencionalidad humana $y$ presenta la experiencia concreta humana mediante una secuencia de eventos en tiempos y lugares determinados. "En este caso, el investigador realiza un análisis narrativo y piensa con los relatos, es un relator de historias y las mismas historias proporcionan el análisis y la teorización" (Bianco, 2012, p. 64).

Por su parte, el pensamiento paradigmático permite explicar los sucesos del mundo físico y sus causas mediante la clasificación de los individuos y relatos bajo un concepto o categoría. Esto último se entiende como el conjunto de atributos comunes que comparten individuos (Bolívar, 2002; Polkinghorne, 1995). Como puede observarse, el acto de relatar implica en cierta forma una nueva alianza entre ciencia y relato (Bruner, 2003, p. 142), entre el pensamiento racional y el narrativo.

Con el fin de establecer la validez o credibilidad y la fiabilidad o transferibilidad se acudió a la triangulación. En cuanto a la validación del respondiente o verificación por parte del mismo participante, se mantuvo comunicación por correo electrónico para cotejar detalles que requerían precisión. Finalmente se realizó el proceso más importante: la restitución al campo. Se enviaron por correo electrónico dos versiones del relato para conocer si el informante coincidía con el texto elaborado o deseaba precisar o ampliar algunos detalles. Esta etapa del proceso metodológico es una respuesta a los detractores de la investigación narrativa pues, contrariamente a quienes la consideran subjetiva, cuando los participantes realizan una lectura de los relatos elaborados, les conceden objetividad. La objetividad está determinada por el hecho de que los participantes nos indican qué es propio de su vida, qué es ajeno, qué es impreciso o puede ampliarse. Lo expresado por Molano (1998), al reflexionar en torno a su historia con las historias de vida, es igualmente válido para los relatos de vida:

[p] ara la gente que la inspira, para la gente que nos cuenta la historia, que nos concede las entrevistas, que nos da la información, se trata de un espejo reestructurado, y nada produce más conciencia que verse uno tal cual es. Creo que esta es una gran ventaja y una virtud de la historia de vida. Es la toma de conciencia por parte de la gente inmediata, y también por parte del investigador. (p. 105).

\section{Resultados}

Puesto que se optó por la modalidad narrativa en el análisis de los datos, empezamos por recordar que un relato de vida constituye un conjunto integral, tejido por un narrador -en este caso, la investigadora- a partir de las narraciones y reflexiones de un informante -Yesid- y de su identificación o satisfacción con lo recopilado por el narrador. En cuanto conjunto integral, tejido por un narrador, se espera que el lector interprete el relato como un todo, como una unidad con sentido. En el relato que se presenta a continuación se recoge ese proceso de construcción de una identidad del participante como docente y escritor, lo cual da sentido como totalidad y coherencia a la narración de sus propias experiencias. Para su lectura, precisamos las siguientes convenciones:

Texto en itálicas: Lo expresado por el informante, presentado en forma literal.

Texto en negrilla: Muestra el énfasis en el tono de voz empleado por el participante.

[texto]: Aclaraciones insertadas para asegurar la claridad de lo expresado por el participante.

...: Pausas en la comunicación.

[...] Omisión de una parte del texto que sigue, con el fin de dar continuidad a las ideas que ilustran el asunto.

Subrayado: Se han marcado así los puntos de inflexión en el relato. Estos se entienden como puntos de cambio en la línea de vida o trayectoria del sujeto, hechos que generan giros generados por una situación o hecho. 


\section{El relato de Yesid: en busca de una voz propia}

Creo que en eso [la mecánica de la escritura académica] ya tengo cierto conocimiento ... pero aún siento que estoy en el desarrollo de mi voz, de mi propio estilo ... no sé si eso lo alcanza uno a hacer en el ejercicio académico o no. Lo siento más propio del ejercicio de escritura en la literatura. Uno sabe cuál escritor es porque tiene un estilo, una voz propia; en cambio en el ejercicio de escritura académica a veces uno no distingue quién está hablando. (Entrevista, 27 de enero de 2014).

Yesid tiene 40 años. Nació en Villeta (Colombia), una ciudad pequeña, ubicada a 91 kilómetros de Bogotá. Es licenciado en filología e idiomas - inglés (1999), magíster en docencia (2007) y especialista en entornos virtuales de aprendizaje (2012). Su experiencia docente de 20 años incluye tres institutos de lenguas y seis universidades colombianas, impartiendo cursos de lengua inglesa, didáctica, investigación educativa y entornos virtuales. En los últimos diez años ha trabajado principalmente en programas de formación inicial de docentes y de maestría. Asiste con regularidad a cursos de actualización, conferencias y congresos y también participa como conferencista.

Su experiencia profesional incluye, además de sus trabajos de grado para obtener los títulos de licenciado y magíster, su participación como investigador en cuatro proyectos sobre pedagogía de la lengua inglesa, lenguas en general, bilingüismo, formación docente y temas educativos de interés institucional. Ha publicado 17 artículos en revistas editadas en su mayoría en Colombia, un capítulo de libro, un texto en memorias de un evento local y dos notas cortas en el boletín de la Asociación Colombiana de Profesores de Inglés (AsOcopI).

Yesid estudió en un colegio público, donde la profesora de inglés marcó su interés por el área de las lenguas debido a que trabajaba mucho con canciones e historias. Además, resaltaba a sus estudiantes los beneficios de dominar el inglés y el francés, pues les brindaba la posibilidad de ver otros mundos. Luego de obtener resultados sobresalientes en las pruebas del Icfes, ${ }^{2}$ decidió que estudiaría trabajo social o algo relacionado con las ciencias humanas. Así, ingresa a una universidad pública en Bogotá, a cursar la licenciatura en filología e idiomas, con especialidad inglés.

Yesid experimentó dificultades para responder a las altas exigencias de la licenciatura: sentía que había compañeros de estudios con muy buen nivel de lengua y no sabía si era lingüista o docente. Con el tiempo, termina encontrando un lugar cómodo en los discursos de las dos disciplinas; disfruta el campo de la lingüística y le gusta también el discurso de la pedagogía y de la didáctica. Antes de terminar la licenciatura se interesa más en la docencia; empieza a trabajar en las mañanas en un instituto de idiomas y en las tardes, en otro. Pero si bien percibía la comodidad de un buen salario, opta por la culminación de sus estudios y reduce las horas de trabajo para dedicarle más tiempo a la elaboración del trabajo de grado, requisito exigido en Colombia para obtener su título como licenciado. Al tiempo que avanza en él, ingresa a un reconocido instituto de lenguas, a trabajar primero con niños y luego con adultos.

El acompañamiento de su directora de trabajo de grado, una reputada lingüista, incidió no solo en la culminación de este, sino también en su interés por la escritura.

Recuerdo que [...] me decía: “¿Dónde está el escrito?”. El trabajo me distraía un poco pero también me quitaba días. Hasta que ella me dijo: “ $\mathrm{No}$, ya no! O usted escribe esa tesis [trabajo de grado] o ya no soy su directora.... Ella había hecho conmigo un trabajo muy juicioso. Me había ayudado muchísimo y creo que eso marcó también un poco mi aventura para incursionar en la escritura.

No obstante, lamenta no haber respondido a la invitación de su asesora a organizar un artículo que podría presentar a la revista Forma y Función, editada en la Universidad Nacional de Colombia.

2 Prueba nacional que presentan los estudiantes al culminar la educación secundaria (grado $11^{\circ}$ ) y que incide en la obtención de un cupo en la universidad. Antes se le conocía como Examen Icfes y ahora se denomina Saber. El examen es administrado por el Instituto Colombiano para la Evaluación de la Educación (Icfes). 
Reconoce que le faltó convicción en sus capacidades para resumir su trabajo de grado en un artículo y, como el instituto de lengua inglesa donde laboraba no le exigía producción escrita, no sentía la importancia de un producto como ese en su labor profesional. El hecho de que allí le exigieran principalmente preparación en metodología y en diseño de materiales, así como las múltiples labores del día tras día, no le dejaban ver con claridad que había una faceta que debía o podía seguir trabajando: el desarrollo escritural de lo que se lee o de lo que se hace en el salón de clase. Considera que, probablemente, esa es una de las razones por las que los docentes no escribimos.

Y ahí quedó la historia de esa tesis [trabajo de grado] porque ella me pidió que yo publicara en Forma y Función y yo ... de visión corta y por estar metido en el trabajo no fui capaz de aprovechar esa oportunidad y ya, cuando la iba a aprovechar, la profesora ya no laboraba en la universidad.

En su infancia, Yesid empezó a plasmar su curiosidad por la escritura en historias que registraba, en formato de película o de guion, en folios que acumulaba, revisaba y editaba con regularidad.

Lleguéa tener un montón de hojas [folios] y a míme gustaba ese ejercicio de sentarme a escribir y escribía una cosa y al día siguiente o a la semana siguiente las retomaba y decía: "Eso está mal". La retomaba y me gustaba más y volvía y la escribía. De ahí surgió un poco la curiosidad por escribir. Nada académico, pero era la curiosidad por escribir.

Yesid recuerda con aprecio la influencia de docentes exigentes, quienes también le mostraron valoraciones positivas por sus esfuerzos y pautas para la mejora. A medida que avanza en sus estudios de licenciatura encuentra docentes que le exhortaron desde las particularidades de las asignaturas que impartían en la licenciatura: literatura, pedagogía y lingüística. En especial, recuerda que

dos docentes en la universidad me permitieron ver la escritura desde otra perspectiva, o sea ... en el colegio era un interés por hacerlo de una manera libre, espontánea y en la universidad fue como "me decido a hacerlo, hágalo bien. Hay una forma de escribir, respete las estructuras, haga planteamientos lo más lógicos posible, organice". Ellos dos fueron vitales para pensar que era posible hacerlo, que no era tan complicado.

Posteriormente, cuando cursa la maestría en docencia, una profesora de pedagogía aconseja a los estudiantes que consideren publicar los trabajos que se producen a ese nivel. Los exhorta a aprovechar las semillas que allí se plasman.

Su primer artículo de reporte de investigación se publicó en 2008, en Profile. Reconoce que, si bien veía difícil lograr publicar en una revista que se reconocía como muy exigente, asumió el reto de resumir la investigación reportada en su tesis de maestría, en un artículo. Fue un proceso arduo y riguroso, durante el cual se sintió animado, ya que recibió retroalimentación muy puntual que, al cabo de varias revisiones, le permitió lograr la meta. "Fue un voto de confianza de las personas [pares evaluadores, editora] que me animaron a mejorarlo, a no desistir; que reconocieron [en el artículo] cosas interesantes ....

Yesid distingue varias huellas que dejó la publicación de su primer artículo de investigación: un logro personal, en tanto publicó un artículo en una revista de prestigio; un logro profesional, evidente en el reconocimiento de sus competencias escriturales y en la constatación de que hay pares académicos interesados en su trabajo; y el poder ser competitivo, puesto que a nivel universitario pesa mucho el hecho de haber publicado en revistas prestigiosas.

Primero, descubrí que sí tenía las herramientas y las habilidades para poder escribir un artículo que entrara en esta revista reconocida. Y segundo, me hizo dar cuenta de ese otro: como miembro de la comunidad escribo no solo para contar lo que he hecho de manera apropiada, sino que contribuyo a la comunidad para que haya una discusión, un avance. [...] Además me muestra que hay una comunidad que quiere leer, que necesita ... y que sí vale la pena escribir...

Yesid ha podido constatar el efecto inminente profesional de haber publicado en Profile. 
Cuando empecé a darme cuenta de personas que me decían: “Ay!, yo leí tu artículo”, o “;ah!, yo leí tal cosa ... Salió en tal revista”. Si bien ese artículo no es el más citado, sí es de los más leídos ... lo han leído colegas de diversas partes del mundo [...]. Ese primer ejercicio serio de escritura me permitió entender que hay una comunidad y una responsabilidad ética frente a ella pues hay una huella que queda allí [en el texto].

A su vez, estos logros le han permitido conocer otras personas, presentar en eventos y emprender otros proyectos escriturales.

Sus cuatro primeros artículos contenían reflexiones metodológicas y se publicaron en un rango de dos años, comprendidos entre 2006 y 2008. Los dos primeros versaron sobre los profesores de lenguas como investigadores y los subsiguientes, sobre la inteligencia y el éxito en el aprendizaje de lenguas.

Así, después de esa experiencia con Profile, Yesid percibe con más claridad qué se espera de un autor de artículos. Progresivamente ha ido buscando otros escenarios para divulgar sus escritos. Ha logrado publicar en otras revistas prestigiosas, editadas en otras universidades colombianas y en México.

Sus reflexiones respecto al papel de la escritura en la carrera profesional lo llevan a concluir que el acto de escribir permite que la cognición del docente se vaya constituyendo y pueda ser referente para otros.

Terminar escribiendo en revistas con cierto nivel de exigencia, pues puede surgir del propio trabajo formal de una tesis o de reflexiones que como docente o como estudiante uno hace y que si en un principio se ven como que me apasionen, como que me gustaría profundizar, pues de ahí sale el trabajo.

$\mathrm{Su}$ trayectoria como escritor también le ha brindado seguridad para dirigir trabajos de grado y siente que, en últimas, el autor empieza a replicar lo que alguien más le ayudó a hacer. De manera informal se vuelve editor, asesor, y confía que después se siga replicando la práctica de apoyo mutuo.

No obstante, ha experimentado el rechazo en dos ocasiones. En un primer intento por publicar, envía a una revista de Argentina un artículo producto de una reflexión presentada en una asignatura de la maestría que cursaba. Publica ese texto en internet $\mathrm{y}$, al cabo de un tiempo, recibe invitación para enviar una versión más desarrollada del escrito, a una revista electrónica especializada en educación en el ámbito latinoamericano. En 2012 presentó otro trabajo a Profile, pero fue rechazado. Reconoce que el texto era muy ambicioso, muy amplio y, aunque admite los motivos por los cuales no se aceptó en primera instancia, esperaba que fuera aprobado luego de enviar los ajustes requeridos.

Piensa que, aunque el rechazo es una posibilidad, nunca es bueno; genera sensaciones de frustración, de sorpresa e incertidumbre, máxime si ya se ha logrado publicar en otras revistas científicas o si se percibe que los puntos señalados por los evaluadores no son totalmente afines. Luego siguió la recomendación de uno de los evaluadores y de la editora de Profile, en el sentido de revisar el trabajo y enviarlo a otra revista, donde fue aceptado. Esto le muestra que como autor o como escritor se debe saber lidiar con el rechazo. Considera que el rechazo es parte normal del proceso; sirve para revisarse, para repensarse y "nos permite ponernos en los zapatos del otro": de nuestros estudiantes, de los colegas que nos piden que evaluemos sus artículos.

Evidentemente el rechazo nunca es bueno. La sensación es frustrante ... tengo que ser honesto: cuando yo recibí la primera evaluación dije: "No, sí". Es obvio [reconoce que hay asuntos para revisar] ... pero cuando pasa el segundo momento de la publicación y ya me dicen "No ... no se publica" ... La sensación fue de sorpresa. Yo ya había publicado [en otras dos revistas editadas en Colombia y clasificadas en niveles altos, como Profile], entonces que me dijeran que no fue como “ $\mathrm{OOh}$, por Dios!”. La primera sensación fue de sorpresa. La segunda fue de un poco de frustración porque ... traté de hacer los cambios que me sugirieron los evaluadores.

Los avances en sus publicaciones le han brindado a Yesid herramientas para aceptar participar como evaluador, primero, de un texto enviado por una universidad norteamericana y luego de revistas. Actualmente es par evaluador de cinco revistas, editadas en Colombia. Realiza esa labor con la convicción de que el propósito central del proceso 
editorial de una revista es, además de publicar, crear una comunidad académica. Yesid opina que una de las grandes misiones de un evaluador de revistas indexadas es promover la colegiatura. El evaluador de artículos debe ir más allá de lo instrumental; debe pensar en cómo se adapta a los contextos de una comunidad joven, académicamente hablando, para que alcance excelencia en la escritura. Remarca que "si yo soy un poco más conocedor de la teoría o se me facilita más la escritura, mi intención debe ser ayudarle a ese colega ... que está necesitando esa ayuda ... invitar, animar a perseverar ... para eso es el evaluador."

Luego de dos años, Yesid relee algunas de sus reflexiones registradas en las primeras entrevistas realizadas en 2014 y señala:

Al leer la primera versión de mi relato de vida, que me compartiste, sigo pensando que esa primera experiencia escritural me hizo entender que la persona que te evalúa es un acompañante; es un colega que te permite pulir. Yo tuve la muy buena fortuna de contar con una persona que, además de paciente, me persuadió a no desistir, a hacer ajustes ... Yo creo que a veces el evaluador puede caer en la falsa idea de que su papel es solamente mirar si un texto cumple con unos parámetros o no y punto.

Yesid resalta que el evaluador

juega un papel importante: es una voz crítica que a la vez anima y aconseja. Entendí que el propósito de esa persona con quien yo estaba en un diálogo era lograr que el producto cumpliera, pero que además yo me animara a no dejar esa posibilidad de desarrollo profesional-porque en últimas lo es [ajustar el texto y publicar]-.

El hecho de ser escritor y docente investigador tiene varios sentidos para la carrera profesional de Yesid. En primer lugar, brinda la posibilidad de cuestionar la propia práctica; en segundo término, permite encontrar en la escritura un canal, un espacio de poder para compartir lo que se indaga, lo que se piensa; finalmente, nos ubica en una búsqueda constante de la excelencia, pues el escribir exige ser más juicioso.
Al preguntarle “¿Cómo te ves hoy como escritor?”, Yesid agrega:

Creo que a todos nos han dicho "tú eres pedagogo, profesor, investigador ... agente de cambio", pero no "itú eres escritor!". Creo que hace falta ganar conciencia ... Ahora ¿por qué no me veo del todo como escritor? Tal vez es que en este momento en nuestro país ese rol de escritor no se le da de entrada al profesor, al investigador. Creo que uno lo ve como incidental: yo escribo como resultado de mi investigación, como algo anexo, pero no como una situación que desarrollo a conciencia, de manera sostenida a lo largo de la carrera profesional ...

$\mathrm{Al}$ realizar una retrospectiva de su trayectoria como autor, Yesid concluye que la escritura es un espejo que le permite mirarse, exigirse más o retomar asuntos. "La escritura ha sido esa posibilidad de verme, mejor dicho, de leerme”. Es también "un espejo. Es mirarme a mí un poco y exigirme más o retomar cosas que había dejado."

La escritura responde a momentos históricos de su vida como docente; en cada uno de ellos manifiesta su progreso, su crecimiento personal y profesional y la forma como interacciona con la comunidad académica. Ahora siente que ha evolucionado como escritor en varios sentidos: es un poco más crítico y asume posiciones; es más arriesgado, menos temeroso; siente mayor posicionamiento como autor al momento de escribir; aborda sus escritos con un mayor grado de profundización, y busca planteamientos diferentes.

Yesid lleva casi veinte años de ejercicio académico juicioso y, si bien ya no se siente un escritor novel pues ha publicado varios textos,

el rótulo de escritor me parece grande, gigantesco. $Y$ sin embargo ... la labor de escritor es parte de lo que hacemos todos los profesores. [...] Sin embargo hemos caído en la trampa de creer que solo somos escritores cuando nos publican ... Creería yo que hay un trabajo interesante para hacer en la formación docente de pregrado, de posgrado, de reconocernos como escritores.

Yesid se proyecta en el futuro como escritor académico y su meta es publicar un libro, para lo 
cual se ha trazado un lapso de cinco a diez años. En 2014 lo proyectaba como un ejercicio individual. Sin embargo, pasados dos años, lo percibe

más como un ejercicio derivado del diálogo. Lo cual me parece un avance porque generalmente yo veía el libro como "soy yo el que escribo como resultado de mis reflexiones", pero ahora veo más el libro pensado en coautoría, como resultado de colegaje, de la reflexión en conjunto con estos colegas que me han acompañado [en proyectos de investigación]. La intención es que en ese libro se decante lo que hemos hecho.

Yesid considera que los artículos científicos tanto de reflexión como de investigación son importantes, valiosos; y se propone continuar publicando. Pero el libro es un llamado personal, un espacio donde podría manifestar quién es uno como docente, su propio crecimiento y la complejidad de nuestra labor.

Un libro debe ser un resultado de ese camino que empieza con artículos, con ponencias o conferencias y memorias ... que décuenta no solo de los resultados de un proyecto de investigación sino también de reflexiones, de posicionamientos propios ... que registre contrastes ... De alguna manera, me permitirá dar una mirada a mi carrera profesional. Podré preguntarme: veinte años después, ¿qué inicié?, ¿quéhe hecho?, ¿quéveo para mi país y para mí mismo? Y en la medida de lo posible contribuir a lo que se requiera, $y$ no necesariamente dedicarnos a replicar lo que otros han dicho. Creo que la idea, aunque ambiciosa, es trascender, hacer nuestros propios planteamientos, teniendo en cuenta el país, la evolución, nuestros propios devenires, presentar la discusión de lo que hemos visto (toma de decisiones, agenciamiento, apropiación, agendas de investigación).

En este momento de su vida profesional Yesid estima que, aunque domina la mecánica de la escritura y los requerimientos de las revistas indexadas, aún está buscando su voz, su postura, su propio estilo y su lugar en los campos del saber propios de su profesión.

Creo que un peligro del sistema actual y en el cual nos movemos los académicos es caer en la trampa de que el ejercicio escritural es casi una fábrica, entonces uno debe fijarse metas para cumplir con la publicación de un número de artículos, de capítulos. Es respetable...
Pero lo esencial es preguntarse: ¿Qué me queda a mí después de escribir dos o tres artículos en un año? ¿Ahí estoy reflejado yo? Creo que a veces uno logra adquirir la comprensión de las bases del ejercicio escritural, pero no logra reflejarse en ese producto.

Por eso se pregunta:

¿Qué identifica a Yesid en la posición de voces en el contexto académico del país, del mundo? ... Y obviamente eso no quiere decir que vaya a descubrir algo totalmente novedoso, pero sí que se perciba en esa producción académica una apropiación y una construcción del investigador. Así se percibe al leer a varios colegas, a quienes identificas cuando los lees y uno logra verles algunos matices... su postura respecto a un asunto determinado.

Yesid espera desligarse de los referentes locales que admira: otros académicos, otros colegas. Probablemente vea eso más claro cuando decida estudiar un doctorado; pero aún si no lo hace, siente que está transitando por ese camino. "Creo que también nace un poco en el momento del doctorado. Eso también es un impulso grande al ejercicio escrito; pero con doctorado o sin doctorado siento que estoy en ese camino."

\section{Conclusiones e implicaciones}

Contar las vivencias de los sujetos, interpretar los hechos y acciones que relatan y ensamblarlos en un relato constituyen una forma distintiva de investigación y un reto en tanto las narrativas del informante $y$ del investigador se funden en un producto, en un texto, para comprender una realidad. En el relato de Yesid se buscó captar la riqueza y detalles de los significados de asuntos determinados; sus motivaciones como docente-investigador para publicar, sus sentimientos frente al logro de unas metas y a circunstancias de fracaso por cuanto algunos de sus textos fueron rechazados; las huellas que ha dejado la publicación de un primer artículo en una revista científica y sus propósitos académicos.

El análisis de las entrevistas mediante el conocimiento narrativo nos permitió observar "la emergencia del sentido que le confiere la persona" (Arraiz y Sabirón, 2011, p. 17) y constatar que la 
narrativa personal autorrecrea el pasado en el presente y lo proyecta en el futuro. El relato no se centra esencialmente en una descripción del presente. Como sugiere E.M. Bruner (1997), "el pasado, el presente y el futuro no solo se construyen, sino que se conectan en secuencias lineales definidas por relaciones sistemáticas, por no decir, causales" (p. 266). Reconocemos, como bien señala el mismo autor, que la forma como representamos algún segmento de la secuencia está relacionada con nuestra propia concepción del todo, del relato. En nuestro caso, interesaba construir un retrato, por así decirlo, del sujeto que se ubica en el presente como autor, rememora diversos momentos de su vida y reflexiona sobre el momento actual y su proyección en el futuro.

El relato de Yesid revela el carácter único de un caso individual y brinda una comprensión de su particular complejidad e idiosincrasia. Yesid, quien se identifica como docente e investigador, muestra cómo a lo largo de su vida y de su ciclo profesional ha ido construyendo identidades, múltiples y cambiantes (figura 1).

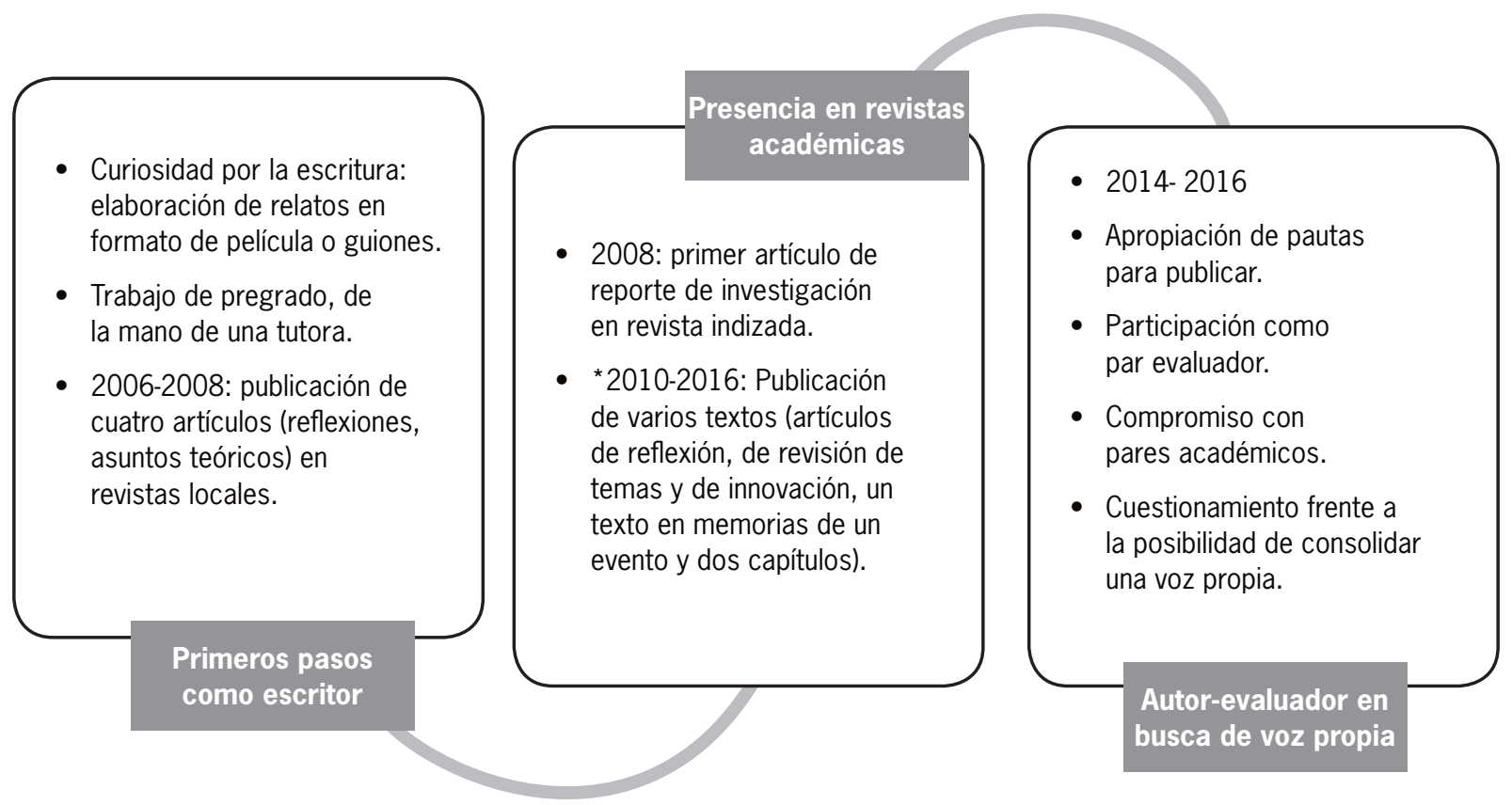

Figura 1. Resumen de la línea de vida del participante como autor

Fuente: elaboración propia.

La línea de vida de Yesid está hecha de "una sucesión de periodos, de acontecimientos, de situaciones" (Bertaux, 2005, p. 41). Como puntualiza el mismo autor, no se trata de una sucesión lineal, "temporal de acontecimientos y de situaciones derivadas de ellos; esta sucesión constituye en cierto modo su columna vertebral [del relato de vida]" (p.37). El relato nos muestra el curso que a lo largo del tiempo ha seguido Yesid, como individuo y también como miembro de grupos sociales, con sus diversos giros o bucles y, por tanto, no puede equipararse a una recta o a una curva armoniosa. En ese recorrido se evidencian el yo y sus identidades -cómo se percibe como autor, su sentir frente al rechazo de uno de sus escritos, qué espera alcanzar en el mundo académico-; su dimensión social -su preocupación por aportar y ser agente de cambio en una comunidad académica-; las relaciones que espera entre el yo docente e investigador, y las culturas o estructuras que 
convergen en el entorno en que labora y, sobre todo, en el ámbito de la publicación en revistas científicas.

El diálogo con el participante deja ver elementos de sus dimensiones personal y académica. Estos se desvelan mientras el sujeto relata hechos sobre su propia vida. No obstante, cabe subrayar que no se pretendió conceptuar respecto a la vida del sujeto primordialmente en términos del lenguaje, es decir, aseverar que a través de sus narraciones se ha identificado su identidad en todas sus dimensiones. Sus relatos y los registros recopilados en algunas evidencias documentales solamente nos acercan al yo y sus identidades en relación con el docente como autor, como escritor. Interesa, ante todo, resaltar el poder de la investigación narrativa para conocer acerca de la vida de los sujetos pues durante la elaboración del relato de Yesid pudimos constatar que, contrariamente al ideal positivista de establecer una distancia entre el investigador y el objeto investigado -el participante-, para garantizar la objetividad en la investigación narrativa y, por tanto, en el relato de vida, los informantes hablan de ellos mismos, sin encubrir su subjetividad.

Conscientes del riesgo de caer en una especie de determinismo o reduccionismo lingüístico y social, reconocemos que al construir el relato del participante encontramos facetas del individuo, sin perder de vista que existen otras formas de experiencia humana que bien pueden incidir en su identidad como autor y cuya exploración trasciende los alcances del presente estudio. Seguramente podríamos ampliar nuestra comprensión del asunto a través de historias de vida, otra alternativa de la investigación narrativa, que implica un estudio de corte longitudinal y el uso de un acervo documental más amplio.

Con todo, constatamos que si bien en los estudios de caso como en el que se enmarca el relato de Yesid no se busca establecer generalizaciones, la narrativa elaborada a partir de sus reflexiones muestra las improntas que ha dejado la publicación de su primer artículo en una revista académica o científica en su ciclo profesional. Probablemente, algunas de sus experiencias, metas y preocupaciones coincidan con otros docentes y con las circunstancias o necesidades locales colombianas o de otros contextos.

De las vivencias de autores como Yesid se derivan asimismo implicaciones no solo para mantener la calidad de la revista sino, lo que es más importante, para empoderar a los autores en las particularidades de la escritura académica en lengua inglesa y animarlos a mantener un rol activo en sus comunidades académicas. Para los docentes de inglés ese es un reto adicional a los que enfrentan actualmente. A su vez, podemos reconocer el papel que cumplen las revistas científicas como generadoras de comunidades académicas y motivadoras para que sus integrantes compartan su saber y desarrollen sus capacidades escriturales. Si, tal como señala la UNESCO (2005), se concibe la sociedad del conocimiento como una colectividad que se nutre de sus diversidades y capacidades, que debe garantizar el aprovechamiento compartido del saber y no reducirse a la sociedad de la información, las revistas y las comunidades a quienes van dirigidas debemos no solo asumir una posición reflexiva y crítica respecto a lo que se produce. Interesa también identificar y actuar para responder a las exigencias que el mundo actual hace al profesorado y de quien se espera una mayor participación en las publicaciones propias de sus disciplinas.

\section{Referencias}

Argüello, A. (2014). La perspectiva biográfica en la investigación educativa: Una mirada a sus antecedentes, tendencias y posibilidades. Estudios Pedagógicos, 40(1), 293-308. doi: 10.4067/S0718-07052014000100018

Arraiz, A. y Sabirón, F. (2011). Una historia inacabada de las historias de vida. Workpaper. Zaragoza: Universidad de Zaragoza.

Barkhuizen, G. y Wette, R. (2008). Narrative frames for investigating the experiences of language teachers. System, 36(3), 372-387. doi: 10.1016/j. system.2008.02.002

Bertaux, D. (1999). El enfoque biográfico: su validez metodológica, sus potencialidades. Proposiciones, 29, 1-23.

Bertaux, D. (2005). Los relatos de vida. Perspectiva etnosociológica. Barcelona: Ediciones Bellatera. 
Bertaux, D. (2009). Life stories. Londres: Sage Publications.

Bianco, I.L. (2012). La investigación biográfico-narrativa o el desafío de descolonizar nuestra mirada. Revista de Educación, 4(4), 89-99. Recuperado de http:// fh.mdp.edu.ar/revistas/index.php/r_educ/article/ viewFile/88/151

Bolívar, A. (2002). “¿De nobis ipsis silemus?”: Epistemología de la investigación biográfico-narrativa en educación. Revista Electrónica de Investigación Educativa, 4(1). Recuperado de http://redie.uabc.mx/ redie/article/view/49

Bolívar, A. y Domingo, J. (2007). Biographical-narrative Research in Iberoamerica: Areas of Development and the Current Situation [112 paragraphs]. Forum Qualitative Sozialforschung / Forum: Qualitative Social Research, 7(4), Art. 12. Recuperado de http://nbnresolving.de/urn:nbn:de:0114- fqs0604125.

Bruner, E.M. (1997). Ethnography as narrative. En L.P. Hinchman y S.K. Hinchman (eds.), Memory, identity, community: The idea of narrative in the human sciences (pp. 264-280). Albany: State University of New York Press.

Bruner, J. (1998). Realidad mental y mundos posibles. Los actos de la imaginación que dan sentido a la experiencia. Barcelona: Gedisa Editorial.

Bruner, J. (2003). La fábrica de historias. Derecho, literatura, vida. Buenos Aires: Fondo de Cultura Económica.

Cárdenas, M.L. (2003). Teacher researchers as writers: A way to sharing findings. Colombian Applied Linguistics Journal, 5, 49-64.

Cárdenas, M.L. (2014). Publishing and academic writing: Experiences of authors who have published in Profile. Profile Issues in Teachers' Professional Development, 16(2), 11-20. doi: 10.15446/profile.v16n2.46144.

Cárdenas, M.L. y Nieto, M.C. (2010). El trabajo en red de docentes de inglés. Bogotá: Universidad Nacional de Colombia.

Cárdenas, M.L., González, A. y Álvarez, J.A. (2010). El desarrollo profesional de los docentes de inglés en ejercicio: algunas consideraciones conceptuales para Colombia. Folios, 31, 49-67.

Connelly, F.M. y Clandinin, D.J. (1990). Stories of experience and narrative inquiry. Educational Researcher, 19(5), 2-14. Recuperado de http://www.jstor.org/ stable/1176100
Cortés, L., Cárdenas, M.L. y Nieto, M.C. (2013). Competencias del profesor de lenguas extranjeras: creencias de la comunidad educativa. Bogotá: Universidad Nacional de Colombia.

Creswell, J.W. (2013). Qualitative inquiry and research design: Choosing among five traditions. $3 \mathrm{a}$. ed. Thousand Oaks: Sage.

Delgado, J.E. (2011). Journal publication in Chile, Colombia, and Venezuela: University responses to global, regional, and national pressures and trends. (Tesis doctoral). University of Pittsburgh, EE. UU. Recuperado de http://d-scholarship.pitt.edu/9049/

Denzin, N.K. (1970). The research act in sociology: A theoretical introduction to sociological methods. Londres: Butterworths.

Departamento Administrativo de Ciencia, Tecnología e Innovación (Colciencias) (2015, diciembre). Modelo de medición de grupos de investigación, desarrollo tecnológico o de innovación y reconocimiento de investigadores del sistema nacional de ciencia, tecnología e innovación, año 2015. Bogotá. Recuperado de http:// www.colciencias.gov.co/sites/default/files/upload/ convocatoria/mediciondegrupos-actene2015.pdf

Departamento Administrativo de Ciencia, Tecnología e Innovación (Colciencias) (mayo de 2016). Documento No. 1601: política para mejorar la calidad de las publicaciones científicas nacionales. (Versión para discusión). Bogotá. Recuperado de http://www. colciencias.gov.co/sites/default/files/upload/paginas/ politica-publindex-colciencias.pdf

Legrand, M. (1999). La contra-transferencia del investigador en los relatos de vida. Proposiciones, 29, 115121. Recuperado de file://C:/Users/MelbaLibia_2/ Downloads/PR-0029-011-3254\%20(2).pdf

Lucio-Arias, D., Rivera Torres, S.C., Tique Ortiz, J., Villarreal, N.F., Lucio, J., Mora, H. ... Garavito Muñoz, M.P. (2015). Indicadores de ciencia y tecnología, Colombia 2014. Bogotá: Observatorio Colombiano de Ciencia y Tecnología. Recuperado de https://www. icesi.edu.co/investigaciones_publicaciones/images/ Documentos/OCyT_Indicadores_2014.pdf

Mendieta, J.A. (2013). Narrative research: An alternative approach to study language teaching and learning. Folios, 37, 135-147. 
Molano, A. (1998). Mi historia de vida con las historias de vida. En T. Lulle, P. Vargas y L. Zamudio (coord.), Los usos de la historia de vida en las ciencias sociales I (pp. 102-111). Barcelona: Anthropos.

Muñoz, G. (2012). El alcance metodológico de las narrativas. En S. Castillo Soler (comp.), Lenguaje y educación: perspectivas metodológicas y teóricas para su estudio (pp. 161-182). Bogotá: Universidad Distrital Francisco José de Caldas.

Organización de las Naciones Unidas para la Educación, la Ciencia y la Cultura (UNESCO) (2005). Informe mundial: hacia las sociedades del conocimiento. Recuperado de http://www.unesco.org/es/worldreport

Oviedo, Y.C. (2009). Competencias docentes para enfrentar la sociedad del conocimiento. Apertura, Revista de Innovación Educativa, 1(11). Recuperado de http://www.udgvirtual.udg.mx/apertura/index. php/apertura/article/view/117/118

Pérez, A.I. (2010). Nuevas exigencias y escenarios para la profesión docente en la era de la información y de la incertidumbre. Revista Interuniversitaria de Formación de Profesorado, 24(2), 17-36. Recuperado de http://www.aufop.com/aufop/uploaded_files/ revistas/127929800810.pdf

Plummer, K. (1989). Los documentos personales. Introducción a los problemas y la bibliografía del método humanista. Madrid: Siglo XxI.
Polkinghorne, D. E. (1995). Narrative configuration in qualitative analysis. International Journal of Qualitative Studies in Education, 8(1), 5-23. DoI: http://dx.doi.org/10.1080/0951839950080103

Polkinghorne, D.E. (2007). Validity issues in narrative research. Qualitative Inquiry, vol.13 (4), 471-486. doi:10.1177/1077800406297670.

Pujadas, J.J. (1992). El método biográfico: el uso de las historias de vida en ciencias sociales. Madrid: Centro de Investigaciones Sociológicas (CIS).

Ricoeur, P. (1995). Tiempo y narración. Vol. I: Configuración del tiempo. Vol. II: Configuración del tiempo en el relato de ficción. Vol. III: El tiempo narrado. Ciudad de México: Siglo XxI.

Ricoeur, P. (1999). Historia y narratividad. Barcelona: Universitat Autònoma de Barcelona.

Sabirón, F. (2007). Métodos de investigación etnográfica en Ciencias Sociales. Zaragoza: Mira Editores. 